\title{
Parameter estimation in multi-antenna system based on iterative adaptive approach
}

\author{
Lihua Lei ${ }^{1}$, Ju Zhou ${ }^{1, a}$ \\ ${ }^{1}$ China Academy of Space Technology, Qian Xuesen Laboratory of Space Technology, 100094, Beijing, China
}

\begin{abstract}
As one of the main means for remote sensing and detecting, synthetic aperture radar is playing more important role in many fields such as country reconnaissance, ocean observation, environment disaster monitoring and military reconnaissance. The synthetic aperture radar system based on multi-antenna technology can achieve high resolution of still image and estimate the motion parameter. The echo model of target is set up for motion parameter estimation and the performance of system parameter estimation is given according to the moving target velocity estimation method based on iterative adaptive approach.
\end{abstract}

\section{Introduction}

As one important feature of modern airborne/ spaceborne radar system, ground moving target indication is of significant value of applications in both military and civil fields. With the extensive application of synthetic aperture radar technology, ground moving target indication based on synthetic aperture radar platform is required to obtain high-resolution images of the static scene of ground, meanwhile to detect moving objects and estimate the motion parameters. In recent years, the ground moving target indication technology based on synthetic aperture radar has obtained prodigious evolvement, but still faces the following challenges ${ }^{[1]}$. Firstly, it is known that the performance of multi-channel clutter suppression depends on system space DOF. However, there are many constraints for space borne synthetic aperture radar system to be installed with more receive channels, such as size, power, load and cost of the platform, which lower the space DOF. Secondly, since the increasing complexity of imaging and moving target detection scenes, especially in cities, mountains and other complex terrain, moving target detection is in face of several practical issues like non-uniform clutter and multipath echoes. Thirdly, increasingly complex electromagnetic environment, including active/passive jamming from enemy, poses critical challenges to image and detect moving targets. Synthetic aperture radar system requires a higher degree of freedom in space to solve these problems. While the way of simply adding more receive channels with limited potential and exploring a new radar system is necessary. Multiple input multiple output is a new kind of radar system which transmits independent waveforms through multiple antennas and receives scene echoes with plurality of antennas independently in order to obtain more spatial sampling in the condition of the same number of antennas ${ }^{[2]}$.

In this article, a mathematical model was built for echoes of static scenes and moving targets in multiple input multiple output imaging mode, based on which a method of moving target parameter estimation was proposed and validated by numerical simulation.

\section{Method of velocity estimation}

During moving target detection process, a set of discrete doppler frequency cells are searched to indicate the presence of moving targets for each resolution cell. Once moving targets are detected within a doppler cell, the estimation of the radial velocity of targets can be acquired. However, the radial velocity estimation of target acquired by this method is rough due to the limited number of doppler detection channels. Accurate estimates need more precise processing method.

\subsection{Rough estimate of radial velocity based on FFT method}

Target's radial motion will cause the azimuth offset in synthetic aperture radar images. In order to correct to the real location, it is necessary to estimate the radial velocity ${ }^{[3]}$

With the clutter suppression, echo of cell to be detected can be expressed as

$$
\mathbf{z}=\sigma_{m} \cdot \mathbf{v} f_{v} \mid \mathbf{n}
$$

The expression of the target's spatial steering vector can be expanded to

\footnotetext{
${ }^{\mathrm{a}}$ Corresponding author: zhouju@qxslab.cn
} 


$$
\mathbf{v} f_{v}=\mathbf{a} f_{v} \text { 必 } \mathbf{b} f_{v}=\left[\begin{array}{cl}
\exp j 2 \pi f_{v} \eta_{T, 1} & \exp j 2 \pi f_{v} \eta_{R, 1} \\
\exp j 2 \pi f_{v} \eta_{T, 1} & \exp j 2 \pi f_{v} \eta_{R, 2} \\
\vdots & \\
\exp j 2 \pi f_{v} \eta_{T, M_{T}} & \exp j 2 \pi f_{v} \eta_{R, M_{R}}
\end{array}\right]
$$

Obviously, the doppler frequency of the echo of target is equivalent to the spatial frequency of target with respect to multiple input multiple output array. When the radial velocity of target is zero, then $f_{v}=0$, which is similar to the situation that signal source is incident from the normal direction of the array. Overall, target's radial velocity estimation is equivalent to the spatial spectrum estimation of array signal, or DOA estimation.

The issue of estimation of target velocity is transformed to DOA estimation of the target with a virtual array. Regular Delay and Sum (DAS) method can be realized by FFT transform ${ }^{[4]}$.

Restricted by the number of spatial sampling points, velocity estimation method based on DAS is defective for the low resolution and higher side lobes. Undoubtedly a more accurate method is needed for the parameter estimation.

\subsection{Accurate estimate of radial velocity based on an IAA method}

Iterative Adaptive Approach (IAA) is a new kind of nonparametric adaptive spectral estimation algorithm, by which higher accuracy spectral estimation can be acquired with only less or even a single snapshot of data. Meanwhile, it offers better robustness for the array of amplitude / phase error and system sampling error ${ }^{[5]}$

In the phases of adaptive moving target detection, velocity range of the moving target is able to be defined roughly. Then the range is divided into $K_{v}$ discrete values. More intensive velocity interval division means higher estimation accuracy, but also higher computational complexity. To simplify the notation $\mathbf{v}_{i} \Delta \mathbf{v} f_{v i}$ and combine the spatial steering vectors of interests into vector in form as

$$
\mathbf{A}=\left[\begin{array}{llll}
\mathbf{v}_{1} & \mathbf{v}_{2} & \ldots & \mathbf{v}_{K_{v}}
\end{array}\right]
$$

Assuming corresponding response function of velocity $f_{v i}$ is $\xi_{i}=; f_{v i}=\sigma_{m, i} e^{j \xi_{i}}$, corresponding response functions of all velocity that interested in are represented also in vector form as

$$
\beta-\left[\begin{array}{llll}
\beta_{1} & \beta_{2} & \cdots & \beta_{K_{v}}{ }^{-\mathrm{T}}
\end{array}\right.
$$

After clutter suppression procession, echo of the cell to be detected is expressed as

$$
\mathbf{z}-\mathbf{A} \beta+\mathbf{n}
$$

IAA solves 3 with weighted minimum variance method in which minimum cost function is

$$
\arg \min \left\|\mathbf{z}-\beta_{i} \mathbf{v}_{i}\right\|_{Q_{i}}^{2}
$$

The above estimation process is achieved with iteration. Generally, IAA use period spectrum estimation as the primary iteration .The process is shown in Table 1.
Table 1. Iterative process of IAA algorithm

Initialization $p_{i i}=\frac{1}{K_{v}^{2}}\left|\mathbf{v}_{i}^{\mathrm{H}} \mathbf{z}\right|^{2}, \quad i=1,2, \ldots, K_{v}$

Iteration

$$
\begin{aligned}
& \mathbf{R}-\mathbf{A} \mathbf{P} \mathbf{A}^{\mathrm{H}} ; \\
& \beta_{i}-\frac{\mathbf{v}_{i}^{\mathrm{H}} \mathbf{R}^{-} \mathbf{z}}{\mathbf{v}_{i}^{\mathrm{H}} \mathbf{R}^{-} \mathbf{v}_{i}} ; \\
& p_{i i}=\left|\beta_{i}\right|^{2} ;
\end{aligned}
$$

Stop Iteration: Reach the set number of iterations, or meet the set convergence criteria;

End

\section{Simulation of scene echo}

Verifying the validity of method of moving target detection requires the clutter data of ground scene. However, it is difficult to obtain measured data from multiple input multiple output system in practice. Weighting scattering coefficient with two-dimensional impulse response of the synthetic aperture radar system by two-dimensional frequency domain method is able to be realized quickly with FFT in two-dimensional frequency domain ${ }^{[6]}$. Depending on the specific relationship of transmitting and receiving echo signals between different combinations, a method for the fast generation of frequency domain of scene data in multiple input multiple output synthetic aperture radar is proposed, which is based on the traditional twodimensional FFT frequency domain simulation method with single-channel raw data ${ }^{[7]}$.

\subsection{Method of Echo Fast Generation in 2D FFT}

Ignoring constant factor, echo of scene is derived from coherent superposition of echo of all scatters ${ }^{[8]}$ and represented as:

$$
\begin{aligned}
S \xi, \eta & -\int_{x} \int_{1} \gamma x_{i}, r_{i} S_{i} \xi, \eta d x_{i} d r_{i} \\
& -\int_{i} \int_{1}^{\gamma} \xi_{i}, H_{i} S_{i} \xi, \eta d \xi_{i} d \eta_{i}
\end{aligned}
$$

Where, $\mathrm{I}_{x}$ and $\Gamma_{r}$ is defined as the coverage area of azimuth and range of Multiple input multiple output beam in ground scene respectively.

By applying two-dimensional Fourier transform on slow time $\eta$ and fast time $\xi$, two-dimensional frequency domain of signal is expressed as

$$
S f_{r}, f_{x}=\int_{\Gamma_{x}} \int_{\Sigma_{r}} \gamma x_{i}, r_{i}\left[\iint S_{i} \xi, \gamma_{i} \exp \quad j 2 \pi f_{r} \xi \quad j 2 \pi f_{x} \eta d \xi d w_{i}\right] d x_{i} d r_{i}
$$

Where, $f_{r}$ and $f_{x}$ represent the range and azimuth frequency respectively, while $S f_{r}, f_{x}$ represents twodimensional frequency spectrum of the echo signal in the scene.

Two-dimensional frequency spectrum of target can be expressed as 


$$
S_{i} f_{r}, f_{x}=G_{0} C_{\xi_{i}, n_{i}} \exp \quad j 2 \pi \eta_{i} P \exp \quad j 2 \pi \xi_{i} Q
$$

Where, $G_{0}, P$ and $Q$ are uncorrelated with the target location but related with $f_{r}, f_{x}$; Conversely $C_{i_{i}, t_{i}}$ is related with target location but uncorrelated with $f_{r}, f_{x}$.

By integrating formula (8) and formula (9), then

$$
\begin{aligned}
& S f_{r}, f_{x}=G_{0} \int_{\Gamma_{x}} \int_{r_{r}}+x_{i}, r_{i} C_{i_{i}, n_{i}} \exp -j 2 \pi \eta_{i} P \exp -j 2 \pi \xi_{i} Q d \xi_{i} d \eta_{i} \\
& =G_{0} F f_{r},\left.f_{x}\right|_{f_{x}=P, f_{r}=Q}
\end{aligned}
$$

Where, $F f_{r}, f_{x}$ is the result of transformed $i x_{i}, r_{i} C_{i_{i}, i_{i}}$ through two-dimensional Fourier transform.

Scene echo signals can be obtained by applying inverse fourier transform to formula (10) and expressed as:

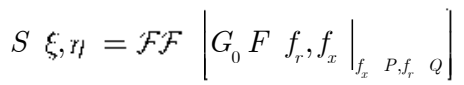

Integration of $\xi_{i}$ and $\eta_{i}$ is realized with 2D FFT and interpolation, that is

$$
\int_{l_{x}} \int_{1_{r}} \because x_{i}, r_{i} C_{v_{i}} \exp \quad j 2 \pi \eta_{i} P \exp \quad j 2 \pi \xi_{i} Q d \xi_{i} d_{\eta_{i}}=\Gamma f_{x}, f_{r}^{\prime}
$$

Where, $F_{s}$ is sampling frequency, $f_{r}^{\prime}$ indicates the position to be interpolated, which is expressed as

$$
f_{r}^{i}=\sqrt{f_{c}+f_{r}^{2}-\left(\frac{f_{x}}{2 v_{p} / c}\right)^{2}}-f_{c}
$$

\subsection{Generation of scene echo}

Two-way time delay caused by the process echo signal transmitted by the $m$ antenna and received by the $n$ antenna is:

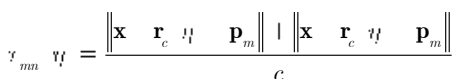

Extends the formula above to

$$
\tau_{m n} \quad \frac{2}{c}\left\|\mathbf{x}-\mathbf{r}_{c} \eta\right\|-\frac{1}{c} \mathbf{u}^{\mathrm{T}} \mathbf{x} \quad \mathbf{p}_{m}-\mathbf{q}_{n} \quad \bar{\tau}+\Delta \tau_{m n}
$$

Where, $\bar{\tau} \triangleq 2\left\|\mathbf{x}-\mathbf{r}_{c} \eta\right\| / c$ is the two-way time delay from referenced phase center of multiple input multiple output array to target point $\mathbf{x}$.

Thus, signal echo of target can be expressed as

$$
\begin{aligned}
y_{m n} \xi, \eta & =u \xi-\tau_{m n} \exp -j 2 \pi f_{c} \tau_{m n} \\
& =u \xi-\bar{\tau}-\Delta \tau_{m n} \exp -j 2 \pi f_{c} \bar{\tau} \exp -j 2 \pi f_{c} \Delta \tau_{m n}
\end{aligned}
$$

Transformed into range frequency domain- azimuth time domain, it is expressed as

$$
y_{m n} f_{r},{ }_{i f}-y_{00} f_{r}, i j \exp -j 2 \pi f_{r} \Delta \tau_{m n} \exp -j 2 \pi f_{c} \Delta \tau_{m n}
$$

Where, $y_{00} f_{r}, \eta$ represents synthetic aperture radar echo of referenced phase center of multiple input multiple output array that transmitted and received with single antenna $^{[9]}$

\section{Simulation and results}

In the simulation scenario, assuming that the number of transmit antennas $M_{T}-2$, the number of receive antennas $M_{R}=8, d_{R}=10 \lambda$ and $d_{T}=M_{R} d_{R}$. There is a moving target with radial velocity of $5 \mathrm{~m} / \mathrm{s}$ in the cell to be detected. Assuming the clutter is completely suppressed after adaptive moving target detection and the system $\mathrm{SNR}=20 \mathrm{~dB}$, Figure 1 shows the estimation results of moving target's radial velocity by DAS and IAA based methods. Figure 1(a) shows the linear representation of normalized amplitude, while Figure 1 (b) demonstrates the normalized amplitude in $\mathrm{dB}$. The result of simulation indicates that radial velocity estimation algorithm based on IAA can offer higher resolution (narrower main lobe) and larger dynamic range (lower ratio of peak value side lobe) compared with radial velocity estimation method based on DAS.

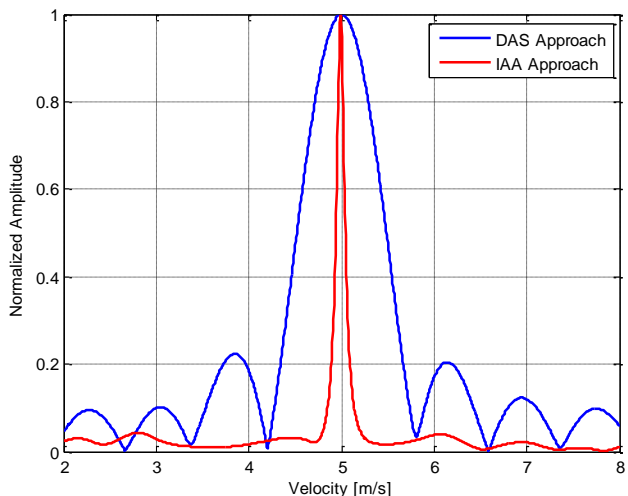

(a)

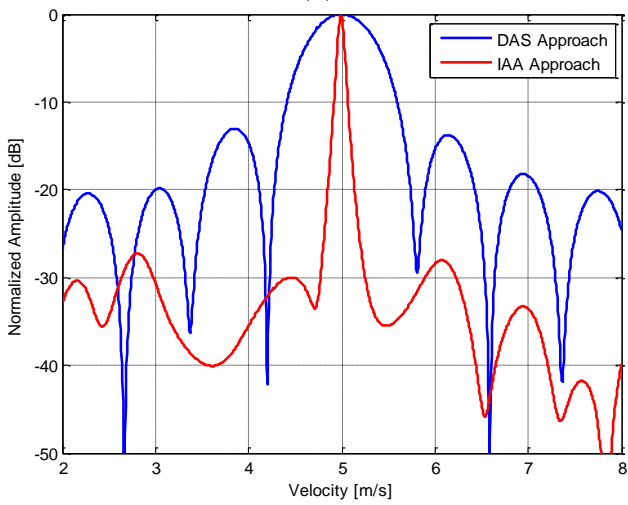

(b)

Figure 1. Comparison of radial velocity estimation methods based on DAS and IAA (a)Liner (b) ) in dB

Assume that there are two moving target points with the same scattering intensity to be distinguished in the resolution cell. The radial velocity are $4.8 \mathrm{~m} / \mathrm{s}$ and 5.2 $\mathrm{m} / \mathrm{s}$ respectively. By using both DAS and IAA algorithm, the results of velocity estimation is shown in Figure 2. The estimation results of DAS in Figure 2 (a) failed to distinguish one target from each other, while estimation results of high resolution spectral estimation algorithm IAA in Figure 2 (b) distinguished two targets apparently. 


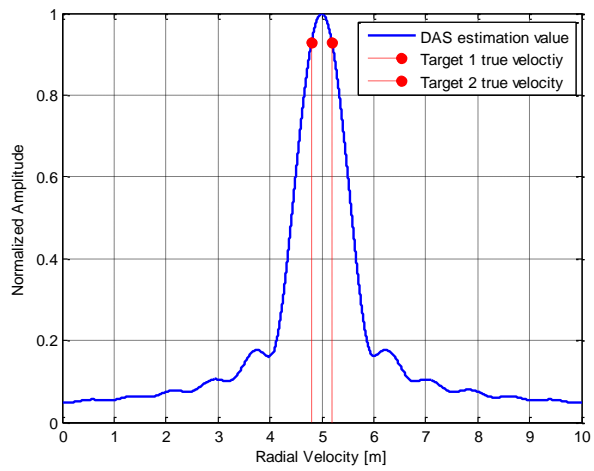

(a)

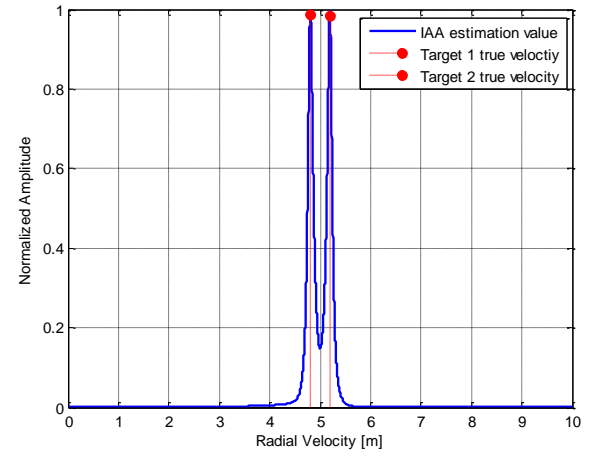

(b)

Figure 2. Comparison of distinguish performance between DAS and IAA method when targets with the same scattering intensity (a) DAS (b) IAA

Assume that there are two moving target points with different scattering intensity to be distinguished in the resolution cell. For target 1, radial velocity is $4.0 \mathrm{~m} / \mathrm{s}$ and the scattering intensity is normalized to 1 . For target 2, radial velocity is $5.0 \mathrm{~m} / \mathrm{s}$, with $-10 \mathrm{~dB}$ lower of scattering intensity than target 1 .

Results of velocity estimation from DAS and IAA algorithm is shown in Figure 3 respectively. Due to the higher side lobe of DAS estimation method, it is difficult to distinguish different targets by DAS estimation since target with higher scattering intensity suppresses target with lower scattering intensity in the same resolution cell. While the side lobe of IAA algorithm is lower and it is possible to distinguish targets with in relatively larger dynamic range of speed.

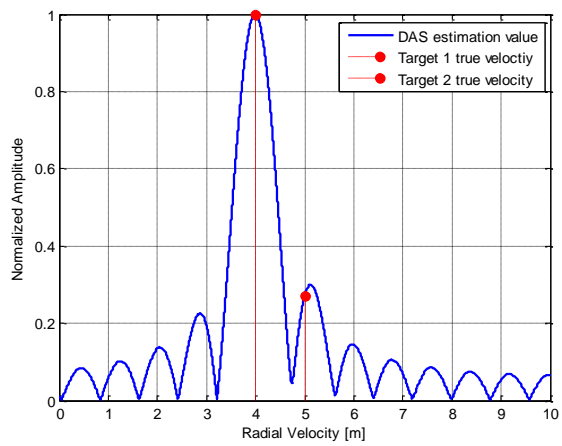

(a)

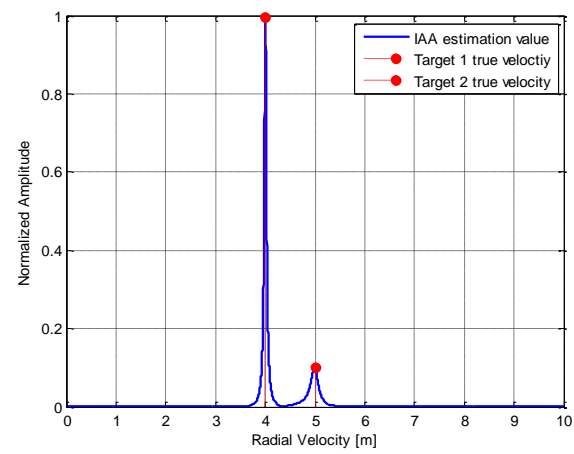

(b)

Figure 3. Comparison of distinguish performance between DAS and IAA method for targets with different scattering intensity (a) DAS (b) IAA

\section{Conclusion}

In this article, a mathematical model was built for echoes of moving targets in multiple input multiple output synthetic aperture radar imaging mode, based on which a method of moving target parameter estimation was proposed and validated by numerical simulation. Results of velocity estimation shows that the iterative adaptive approach is possible to distinguish relatively larger dynamic range of speed.

\section{References}

1. Ender J H G. Proceedings of the International Radar Symposium (IRS), Cologne, Germany, 2007.

2. Ender $\mathbf{J} \mathrm{H}$ G. Proceedings of the European Conference on Synthetic Aperture Radar (EUSAR),Friedrichshafen, Germany 2008.

3. Ender J H G, Klare J. Proceedings of the IEEE Conference on Radar, Pasadena,CA, 2009.

4. Bliss D W, Forsythe $\mathrm{K} \mathrm{W}$, Davis $\mathrm{S} \mathrm{K}$, et al. International Waveform Diversity and Design Conference, Kissimmee, FL, 2009: 118-122.

5. Bliss D W. The 41th Asilomar Conference on Signals, Systems and Computers, Pacific Grove, CA, 2007: 207-211.

6. Kantor J M and Bliss D W. The 43th Asilomar Conference on Signals, Systems and Computers, Pacific Grove, CA, 2010: 1821-1826.

7. Forsythe K W and Bliss D W. IEEE Journal of Selected Topics in Signal Processing, 2010, 4(1): 21 32.

8. Wang W Q. 2011 Fourth International Conference on Intelligent Computation Technology and Automation, Shenzhen, Guangdong, 2011: 608-611.

9. Wang $\mathrm{W}$ Q and Cai J Y. IEEE International Geoscience and Remote Sensing Symposium (IGARSS), Vancouver, BC, 2011: 1662-1665. 